\title{
Surveillance Video Portrait Recognition Preprocessing Technology for Police Actual Combat
}

\author{
Yao Chen ${ }^{1}$, Ao Chen ${ }^{2 *}$, Zhiwei Jiang1, Jianfu Zhong ${ }^{1}$ \\ ${ }^{1}$ School of Information and Electronic Engineering, Zhejiang Gongshang University, Hangzhou, China \\ ${ }^{2}$ Jinhua Public Security Bureau, Jinhua, China \\ Email: *349667647@qq.com
}

How to cite this paper: Chen, Y., Chen, A., Jiang, Z.W. and Zhong, J.F. (2019) Surveillance Video Portrait Recognition Preprocessing Technology for Police Actual Combat. Open Journal of Applied Sciences, 9, 394-402. https://doi.org/10.4236/ojapps.2019.95033

Received: April 24, 2019

Accepted: May 27, 2019

Published: May 30, 2019

Copyright () 2019 by author(s) and Scientific Research Publishing Inc. This work is licensed under the Creative Commons Attribution International License (CC BY 4.0).

http://creativecommons.org/licenses/by/4.0/ (c) (i) Open Access

\begin{abstract}
With the continuous development of technology, face recognition technology has played an important role in police work. In order to get a clear face image, image preprocessing technology is needed. This paper mainly proposes illumination compensation technology and reconstruction technology based on symmetry.
\end{abstract}

\section{Keywords}

Wiener Filter, Logarithmic Transformation, Wavelet Transform, Symmetry, Gradient Descent

\section{Introduction}

Using face recognition technology, police officers can be used to conduct case investigations, determine the route of the suspect, delineate the scope of the suspect's activities, and serialize the case. In the traditional video surveillance mode, the video scouts need to first observe the video around the incident to highlight the suspects, and then use the video relay to highlight the behavior track, finally confirm the identity of the suspect by visiting the important places above the track, involving a large number of police forces and time, and timeliness is not high. The application of face recognition technology can quickly obtain the face of a character from a large amount of video, and compare it with the existing face database to confirm the identity of the person. It can also automatically draw the activity track of the character through the video relay. The use of portrait recognition technology in video surveillance helps to improve the efficiency of identifying with standardized, standardized and programmed opera- 
tions, and provide effective detection of cases. At the same time, it can save the verification time of police officers in the scenes of tracing suspects and dangerous personnel.

At present, many researches are focused on face feature extraction and recognition, but there are few researches on image preprocessing. Jiao Linan [1] et al. proposed a subblock partially overlapping logarithm transformation algorithm for non-uniform illumination images. They propose to use subblocks of a certain size to divide the image into many overlapping subimages, and then perform logarithmic transformation. This algorithm shows good performance and controllability, but the calculation is complex. Zhang Jianming [2] et al. proposed a method for processing partially occluded expression images. According to the "three Chambers and five eyes" structure of human face, they judge whether there is occlusion by vertical integral projection of the eye area, and then carry out symmetrical transformation for the face part without occlusion under the condition of retaining the features of the mouth. Then, $2 \mathrm{~d}$-dct was used to extract facial features, and HMM model was used for training.

\section{The Importance of Image Preprocessing Technology in Face Recognition}

Face recognition technology digitally stores image information for feature extraction, optimization, retrieval comparison and big data operation. Key processes include face acquisition and detection, face image preprocessing, face feature extraction, and face recognition classification.

Due to the interference of light, angle, distance, etc., it is often impossible to directly operate on the obtained original face image. Image preprocessing must be performed on the basis of portrait detection. The main purpose is to eliminate noise in the image, enhance image contrast, obtain a portrait image with appropriate contrast and noise, and allow the image to be cut and analyzed, and the size and the facial features are unified and normalized. Preprocessing operations generally include grayscale, binaryzation, geometric correction, filtering, restoration, and enhancement of face images [3]. In the development of face recognition technology, we are more concerned about how to improve the recognition of faces. The quality of the face image is directly related to the performance of the face recognition system. The face image is used as an input variable of the recognition system, and its definition, edge characteristics and contrast affect the recognition result. External information interference reduces the quality of the image. Image preprocessing techniques can make the image more sharp, or enhance certain information in the image to highlight features. The pixel of the image is changed by a certain operation to achieve the purpose of improving the image quality, and the complex image is converted into a feature vector that is easy for the computer to recognize. The quality of image preprocessing will directly affect the detection rate of face recognition. This paper mainly proposes illumination compensation technology, based on symmetry reconstruction technology. 


\section{Illumination Correction Technology}

Extracting surveillance videos installed in streets or large shopping malls is often affected by lighting, resulting in unsatisfactory picture quality. In some scenes with a wide illumination range, the dynamic image will contain shadows and bright areas, which will adversely affect feature extraction. Uneven illumination can cause high-light or shadows on the face image. Even for the same face, its gray histogram will have a big difference under different illumination. As Figure 1 shows.

In order to solve the influence of illumination to a certain extent, this paper intends to use a combination of logarithmic transformation and wavelet transform. First, logarithmic transformation of the face image is performed using a logarithmic relationship that conforms to the characteristics of the human visual system. The dark part of the image will be better enhanced [1]. Then, the obtained log transformed image is wavelet transformed to obtain LL (low frequency approximation component), LH (vertical edge detail component), HH (diagonal edge detail component), HL (Horizontal edge detail component); then the edge information processing is performed on the high-frequency part different from the previous high-pass filtering, and the edge information is more prominent while removing the noise, the Wiener filtering [4] is performed on the low-frequency component, and then the wavelet image reconstruction is performed, thereby improving The quality of the face image under illumination.

The algorithm flow is shown in Figure 2.
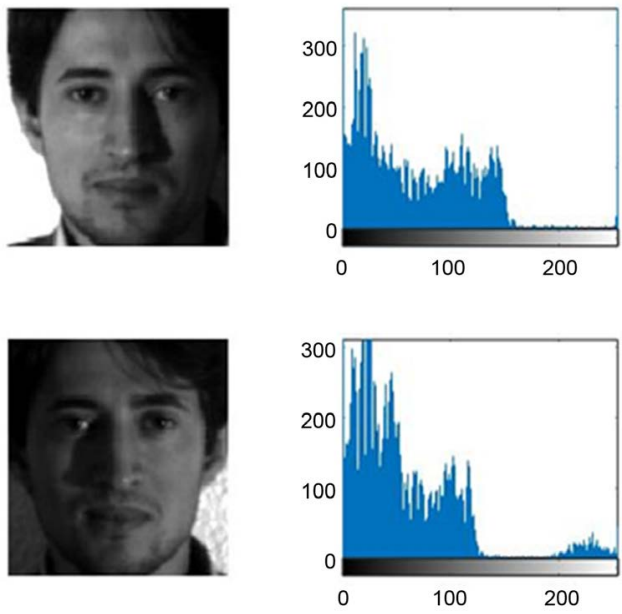

Figure 1. Human face gray histogram under different illumination.

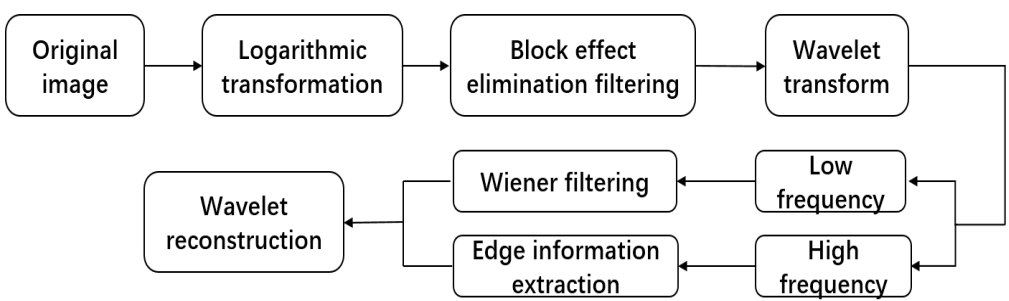

Figure 2. Light correction technology flow. 


\subsection{Logarithm Transformation}

The human visual system is logarithmic to the RGB tristimulus signal. This logarithmic relationship is expressed as follows:

$$
g(x, y)=a+\frac{\ln (f(x, y)+1)}{b * \ln c}
$$

The parameters $a, b$, and $c$ are parameters introduced to correct the curve change; $f(x, y)+1$ is to ensure that after taking the logarithm is still meaningful. Experience has shown that a takeing $0, b$ takeing $\frac{1}{255 * \ln 1.2}$, and $c$ takeing 255 can effectively compensate for light.

The logarithmic transformation is introduced by the visual perception model of Equation (1), which can be described as follows: Let the gray of $f(x, y)$ of the original image be $r$, and become another image $g(x, y)$ by the nonlinear transformation function $s=T(r)$. And the histograms of the two are $P(r)$ and $P(s)$. The global logarithmic transformation compresses the contrast in the high grayscale range, so here we use the POPLE algorithm proposed by Jiao Linan [1] to set the appropriate sliding window size and sliding distance, and perform partial logarithmic transformation of the sub-blocks on the image, and then pass the block. The effect cancellation filter eliminates a small number of block effects produced by partial logarithmic transformation of sub-blocks, improving the effect on contrast in the high gray level range (Figure 3).

\subsection{Wavelet Analysis}

Wavelet analysis is the decomposition of a signal into a series of successive approximation expressions obtained by translation or stretching operations from the original wavelet function. In this paper, using Gabor wavelet transform, Gabor wavelet is very similar to the simple visual stimulation of cells in human visual system. It is sensitive to the edge of image, can provide good direction selection and scale selection characteristics, and is not sensitive to illumination changes. It can provide good adaptability to light changes and extract appropriate human contact information features.

A typical 2-D Gabor function $h(x, y)$ is [5]:

$$
h(x, y)=\frac{1}{2 \pi \sigma_{x} \sigma_{y}} \exp \left(-\frac{1}{2}\left(\frac{x^{2}}{\sigma_{x}^{2}}+\frac{y^{2}}{\sigma_{x}^{2}}\right)\right) \cdot \exp (2 \pi j w x)
$$

Using $h(x, y)$ as the mother wavelet, a series of self-similars can be obtained by performing appropriate scale transformation and rotation transformation on it:

$$
h_{m n}(x, y)=a^{-m}\left(x^{\prime}+y^{\prime}\right), a>1, m, n \in Z
$$

\begin{tabular}{|c|c|c|c|c|}
\hline $\begin{array}{l}\text { Define filter } \\
\text { boundary }\end{array}$ & $\begin{array}{l}\text { Computed } \\
\text { boundary } \\
\text { strength }\end{array}$ & $\begin{array}{c}\text { Filter } \\
\text { switching } \\
\text { decision }\end{array}$ & $\begin{array}{l}\text { Filter strength } \\
\text { decision }\end{array}$ & $\begin{array}{l}\text { Unblock } \\
\text { filtering }\end{array}$ \\
\hline
\end{tabular}

Figure 3. Block effect elimination filtering process. 
where: $\quad x^{\prime}=a^{-m}(x \cos \theta+y \sin \theta), y^{\prime}=a^{-m}(-x \cos \theta+y \sin \theta), \theta=\frac{n \pi}{K} ; a^{-m} \quad$ is a scale factor; $S$ and $K$ is the number of scales and directions; $0 \leq m \leq s-1$, $0 \leq n \leq K-1$. Different wavelet functions can be obtained by changing the values of $m, n$.

\subsection{Wiener Filtering}

Wiener filtering uses the least mean square as the error limit. The goal is to find a convolution function $g(t)$ and get an estimate of $\hat{x}(t)$, where $\hat{x}(t)$ let the square error between $\hat{x}(t)$ and $x(t)$ is obtained minimally, and $y(t)$ is the observed signal and contains noise information (Figure 4).

$$
\hat{x}(t)=g(t) * y(t)
$$

For the four components obtained after wavelet transform, the low-frequency component contains a large number of original image approximation information, which is robust. The Wiener filter is used to process the filtering noise. The three high-frequency components are mainly the edge information of the image, and the stability is poor. Canny edge operator is used for detection. Wavelet processing is performed on the processed components, and the original image is restored.

\section{Reconstructing Face Images Based on Symmetry}

We can know that the face can be regarded as a basically symmetrical structure [6], and this feature can be used to deal with partially occluded face images or uneven light on the left and right half faces. The proposed method is suitable for
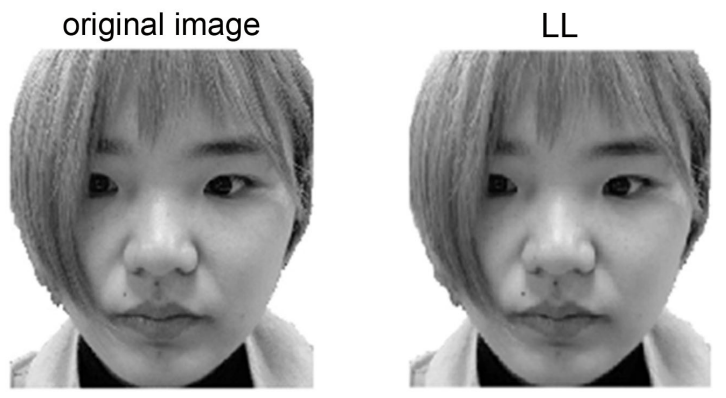

$\mathrm{HL}$

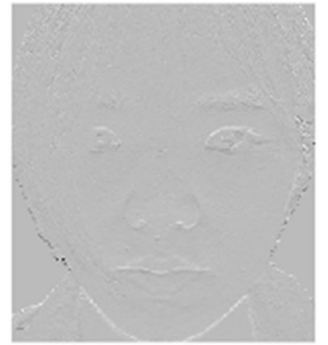

$\mathrm{LH}$ $\mathrm{HH}$
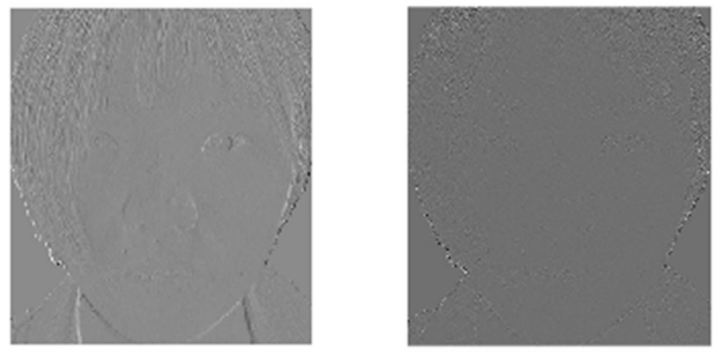

Figure 4. Wavelet transform. 
a positive face or a slight deflection. The method of this paper is to judge whether there is occlusion by integral projection, and determine the center line of the face on this basis, divide the face into four parts of up, down, left and right, and perform gradient descent optimization [7] processing on the upper left and upper right, lower left and lower right respectively then synthesize the face.

\subsection{Integral Projection}

Integral projection is a method of target localization based on gray statistics. Commonly used are vertical integral projection and horizontal integral projection. This paper adds diagonal projection, smoothes the obtained integral map, removes the chopping wave, finds the wave trough, and then judges whether there is occlusion according to the symmetry in the integral graph. As shown in (a) of Figure 5, the face without occlusion has obvious symmetrical structure in the vertical integral diagram. Because the cheeks are in the bright part after binaryzation, as shown in Figure 5, there will be a distinct peak in the horizontal integral map. On the basis of that the face vertical and horizontal are defined as shown in Figure 6.

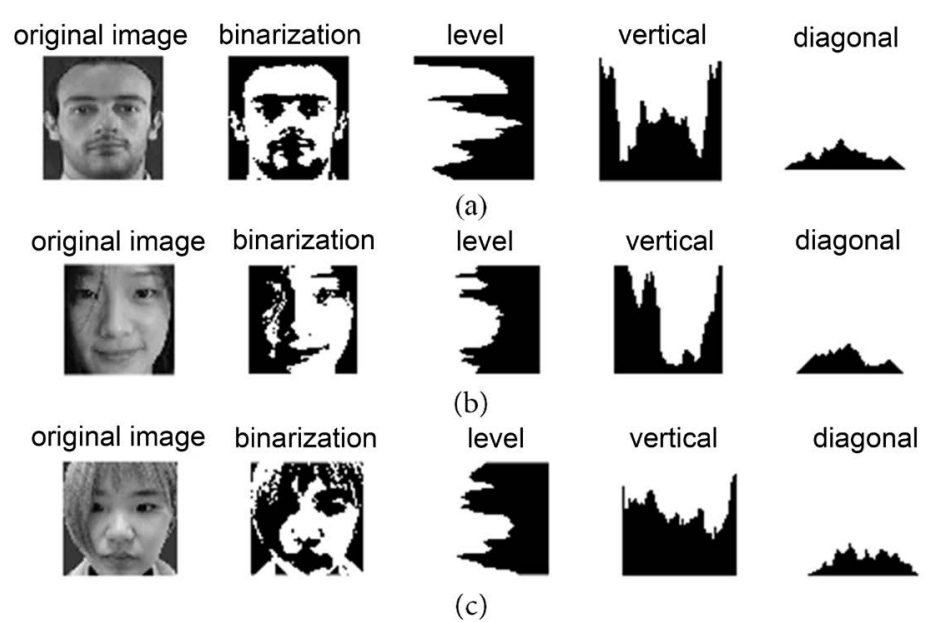

Figure 5. Integral projection.

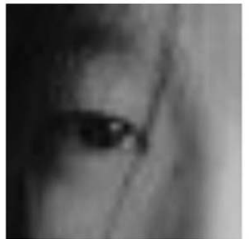

(a)

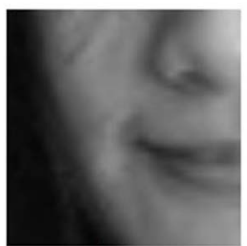

(c)

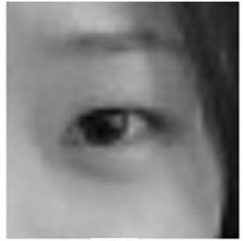

(b)

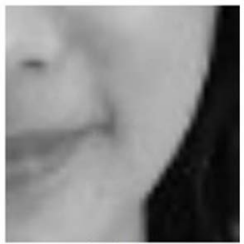

(d)

Figure 6. Face segmentation map. 
After getting the four parts of the original image, we use $A 1, A 2$ to represent the upper left and upper right parts, respectively, with $B 1, B 2$ indicating the lower left and lower right corners. Assuming that the original image can be represented as a $2 \mathrm{D}$ matrix of $m \times n$, where $a$ and $b$ are the coordinates of the center point respectively. Using 1 and 2 to distinguish the upper and lower parts, $A 1, A 2, B 1, B 2$ can be expressed as:

$$
A 1=\left[\begin{array}{c}
F_{1,1} \\
\vdots \\
F_{1, a}
\end{array}\right] A 2=\left[\begin{array}{c}
F_{1, a+1} \\
\vdots \\
F_{1, m}
\end{array}\right] B 1=\left[\begin{array}{c}
F_{2,1} \\
\vdots \\
F_{2, a}
\end{array}\right] B 2=\left[\begin{array}{c}
F_{2, a+1} \\
\vdots \\
F_{2, m}
\end{array}\right]
$$

\subsection{Gradient Exchanging}

Using the gradient descent algorithm, $A 1, A 2$ tend to approximate, with $B 1, B 2$ also tending to approximate. Introducing the $L 2$ norm, the distance function between $A 1$ and $A 2$ can be expressed as Equation (6), and $A 1$ and $A 2$ need to be constantly updated to minimize $L(A 1, A 2)$ :

$$
L(A 1, A 2)=\|A 1-A 2\|_{2}^{2}
$$

The traditional gradient function is

$$
\nabla L(x)=2(A 1-A 2)
$$

Equation (7) is used to update al and a2, which is slow and does not reflect the difference between the two half faces. It has been found that the image with poor illumination or occlusion is dark and the image quality is poor. It is assumed that this part is $A 2$. This paper proposes a new iterative function, which is mainly for the part with poor image quality:

$$
\begin{gathered}
A_{1}^{t+1}=A_{1}^{t}-\alpha\left(A_{1}^{t}-A_{2}^{t}\right) \\
A_{2}^{t+1}=A_{2}^{t}-\alpha\left(A_{2}^{t}-A_{1}^{t}\right)+\left(A_{2}^{t}-A_{2}^{t-1}\right) \int_{0}^{A_{2}^{t}} \frac{1}{2 \pi \sigma} \exp \left(\frac{(x-\mu)^{2}}{2 \sigma^{2}}\right) \mathrm{d} x
\end{gathered}
$$

$A_{1}^{t+1}$ represents the $A 1$ value at the iteration $t+1$, and $A_{2}^{t+1}$ represents the $A 2$ value at the iteration $t+1$, and $\alpha$ is introduced to make the value stable, $\alpha$ is taken $\beta / t$, where is a constant, $t$ is the current iteration number, $\sigma=\frac{A_{2}^{t}-A_{2}^{t-1}}{2}$, $\mu=\frac{A_{2}^{t}+A_{2}^{t-1}}{2}$.

The iterative update stops when the following conditions are met:

1) exceeded the preset number of iterations;

1) $\left\|A_{1}^{t+1}-A_{1}^{t}\right\|<\varepsilon, \varepsilon$ is a very small normal number;

For $b 1, b 2$ does the same (Figure 7 ).

\section{Experimental Analysis}

In order to validate the proposed method, the yaleA face database and the photos taken by ourselves were used for experiments. The yaleA face database contains 


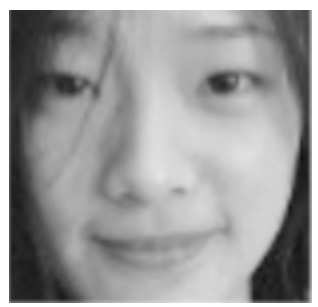

before

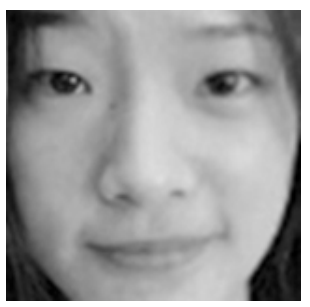

after

Figure 7. Images before and after reconstruction.

Table 1. Recognition rate.

\begin{tabular}{ccccc}
\hline & FIRST & SECOND & THIRD & FOURTH \\
\hline Not processed & $65.32 \%$ & $71.86 \%$ & $72.31 \%$ & $72.35 \%$ \\
After lighting correction & $86.54 \%$ & $84.32 \%$ & $89.12 \%$ & $86.89 \%$ \\
Based on symmetric reconstruction & $70.89 \%$ & $67.32 \%$ & $86.32 \%$ & $84.65 \%$ \\
Fix + refactor & $89.65 \%$ & $93.83 \%$ & $92.65 \%$ & $80.12 \%$ \\
\hline
\end{tabular}

15 people, each with 11 pictures and a total of 165 images. The photos took by ourself included 10 people, 11 photos per person, with different lighting conditions and occlusion pictures. Take 8 photos of each person as training samples and others as test samples (Table 1).

It is seen from experiments that the method of reconstructing images based on symmetry needs to be improved. When processing the deflection angle, deviations will occur, affecting feature extraction, and block effects will occur when the tiles are merged. So further improvement is needed. For face recognition with light corrected images, it has a good effect.

\section{Conclusions}

The two methods proposed in this paper can improve image quality and detection rate to some extent. In addition, the method to find the center line needs to be improved, and 3D streamline model can be introduced in the later research.

The use of science and technology in policing has become unstoppable, which is conducive to case investigation, manpower liberation and time saving. The application of face recognition technology in police affairs can improve the actual combat efficiency of police affairs, promote the development of science and technology, and further serve the actual combat work of police affairs.

\section{Acknowledgements}

Supported by: Provincial key research and development projects in 2018 (2018C01084).

\section{Conflicts of Interest}

The authors declare no conflicts of interest regarding the publication of this paper. 


\section{References}

[1] Jiao, L.N., Sun, Z.Y. and Sha, A.M. (2011) Subblock Partially Overlapping Logarithmic Transformation of Non-Uniform Illumination Image. Computer Engineering and Application, 47, 196-199.

[2] Zhang, J.M. and Zhang, X.C. (2011) A Method for Processing Partially Occluded Expression Images. Computer Engineering and Applications, 3rd Issue.

[3] Guo, Q. (2014) Research on Face Recognition Based on Video Monitoring. Anhui University, Hefei.

[4] Huo, Y.-H. and Fan, W.-Q. (2019) A Face Recognition Method under Complex Lighting Conditions in Coal Mine. Advances in Laser and Optoelectronics, No. 1, 66-67.

[5] Xie, X.D. and Kin, M.L. (2006) Gabor-Based Kernel PCA with Doubly Nonlinear Mapping for Face Recognition with a Single Face Image. IEEE PAMI, 26, 131-137.

[6] Wang, Y.-N. and Su, J.-B. (2013) Symmetry Description and Face Recognition Using Face Symmetry Based on Local Binary Pattern Feature. 32nd Chinese Control Conference (CCC), 26-28 July 2013, Xi'an, 3955-3960.

[7] Li, Y., et al. (2018) Distance Metric Optimization Driven Convolutional Neural Network for Age Invariant Face Recognition. Pattern Recognition: The Journal of the Pattern Recognition Society, 75, 51-62.

https://doi.org/10.1016/j.patcog.2017.10.015 